\title{
Avoidance behavior and ileum motility in monkey during different conditioning schedules pre- and postcingulumotomy
}

\author{
JOAN S. LOCKARD, ELDON L. FOLTZ, RONALD G. HALLER, \\ and ALBERT L. EHLE \\ Department of Neurological Surgery, University of Washington, Seattle, Washington 98195
}

\begin{abstract}
Lever-pulling behavior and ileum motility are simultaneously compared in rhesus monkeys during chronic avoidance conditioning (14 days), before and after either cortical-control or bilateral cingulum lesions. Each day consisted of $12 \mathrm{~h}$ of aperiodic conditioning interspersed with a total of $12 \mathrm{~h}$ of aperiodic rest. The data indicated that lever-pulling and percent ileum motility per avoidance schedule tended to change in the same direction. The cingulum ablations seemed to either augment or reduce both avoidance behavior and percent ileum motility, depending upon the particular monkey. The direction of change in avoidance behavior and percent motility postcingulumotomy appeared to be related to the animal's characteristic ileum response (hypo- or hypermotile) during avoidance conditioning. The results are discussed in terms of the importance of determining individual, baseline emotional reactivity in the assessment of cingulum manipulation.
\end{abstract}

Gut motility is an autonomic phenomenon which may reflect certain emotional influences from the limbic system (e.g., Brady, Porter, Conrad, \& Mason, 1958; Foltz, 1965; MacLean, 1970), and measurement of this parameter may therefore give a means of assessing its involvement in the emotional sphere. The present authors (Lockard, Foltz, Ehle, \& Haller, 1977) have shown that chronic avoidance conditioning affects the daily ileum-motility output of chair-confined monkeys. Most subjects showed either hyper- or hypomotility of gut activity during conditioning (e.g., Figure 1). Moreover, bilateral cingulum ablations tended to reverse the ileum motility changes in these monkeys, while the records of control-lesion monkeys were largely unchanged under identical conditions. The current study reports the avoidance behavior of these same monkeys, preand postcingulumotomy, and subdivides and compares the ileum motility and avoidance responses with respect to the different component schedules used in the conditioning.

\section{METHODS}

\section{Animals and Experimental Design}

Sixteen adolescent (3-5 kg) monkeys (Macaca mulatta) composed equally two main lesion groups and four behavioral subgroups. (The monkeys were the same animals utilized in Lockard et al., 1977; specific motility and avoidance data are reported here.) Each monkey was either an experimental or a yoked behavioral control, and either a bilateral cingulum or cortical-control lesion

This research was supported by National Institutes of Health Grants MH-02895 and NS-04053. E. L. Foltz' present address: Chairman, Division of Neurological Surgery, University of California at Irvine, Irvine, California. animal. The yoked animals were avoidance trained, but had nonfunctional levers during the study proper so they they received the same shocks as their experimental partners. All of the monkeys were on a constant daily ( 0800 to $1000 \mathrm{~h}$ ) food intake schedule of fruit and Purina Monkey Chow, according to

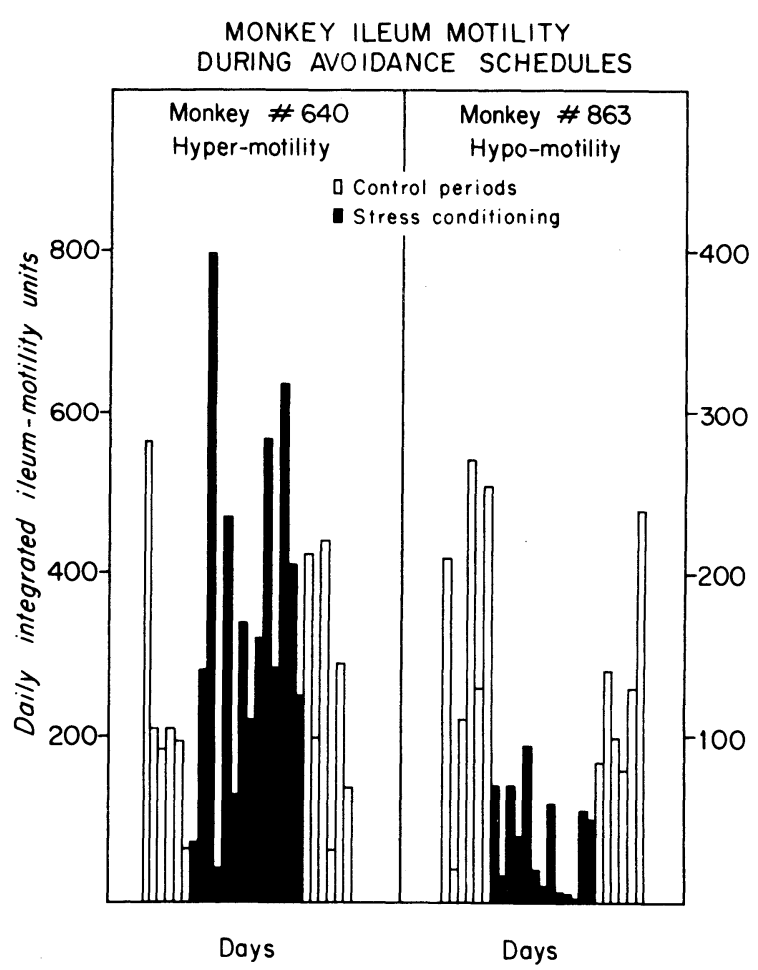

Figure 1. Total daily ileum motility during control periods and stress conditioning (i.e., a combination of avoidance schedules). Stress responses took the form of hyper- or hypomotility. Only prelesion data are shown. 
body weight. They were adapted to primate restraining chairs and housed in an air-conditioned $\left(23^{\circ} \mathrm{C}\right)$ and continuously illuminated (42 fc) laboratory. Each restraint chair was instrumented with a joystick lever at sitting-waist height and a panel of stimulus lights at eye height immediately in front of the animal. All monkeys were trained (Lockard, 1969) to pull the lever to avoid electric shock (Barensten \& Lockard, 1969). Following these sessions, each monkey was administered 1 to 2 days of training similar to the avoidance conditioning of the experiment proper.

\section{Avoidance Schedules}

In the main phase of the study, the monkeys received 14 days of prelesion avoidance conditioning which was replicated after a postlesion recovery period of 7-10 days. Ten different daily programs were utilized so as to minimize animal adaptation to the conditioning (e.g., Dubois \& Natelson, 1978). Each day consisted of $12 \mathrm{~h}$ of aperiodic conditioning interspersed with a total of $12 \mathrm{~h}$ of aperiodic rest. Each schedule was indicated by the illumination of one stimulus light or a combination of the three stimulus lights in the panel on the restraining chairs. Rest was indicated by no lights on.

The four avoidance schedules were active avoidance alone (1); active avoidance plus passive avoidance (4); active avoidance plus unavoidable shock (5); and active plus passive avoidance plus unavoidable shock (7). Active avoidance (AA) consisted of a 10 -mA body shock, for $.5 \mathrm{sec}$ duration, delivered every $20 \mathrm{sec}$ unless the animal reset the shock timer by a lever pull. Passive avoidance (PA) was a low-intensity shock, $.5 \mathrm{~mA}$, to the body and hand for the duration of a lever pull, each time the animal pulled the lever. The occasional aperiodic unavoidable body shock (UAS) was $10 \mathrm{~mA}$ for $.5 \mathrm{sec}$ duration. (See Barensten \& Lockard, 1969 , for additional details of shock delivery.)

Ten different daily programs were statistically equated by counterbalancing (via a Graeco-Latin square, Fisher \& Yates, 1963 , pp. 86-89) the four avoidance schedules such that each schedule, for the same duration, immediately preceded and followed every other schedule in the 14 days of programming. This balancing was restricted by the requirements that (a) one of the four rest periods start at $0800 \mathrm{~h}$ every day and last at least $3 \mathrm{~h}$, the first $2 \mathrm{~h}$ of which the animals were fed; (b) one rest period per day be 5-7 h long; and (c) each schedule occur for a total of $3 \mathrm{~h}$ per day.

A pilot cingulum monkey, No. 715 , in addition to the above four avoidance schedules $(1,4,5$, and 7$)$, received daily passive avoidance alone (2), unavoidable shock alone (3), and passive avoidance plus unavoidable shock (6). Each of the seven schedules for this monkey occurred for a total of 1.5 rather than $3 \mathrm{~h}$ per day. This initial animal was also given 18, rather than 14, days of avoidance conditioning. As a consequence of its performance, only four schedules and 2 weeks of conditioning, preand postlesion, were included in the main experimental design.

\section{RESULTS}

\section{Data Collation}

The raw data were of two kinds: (a) lever pulls and (b) integrated units of ileum motility (Lockard et al., 1977). The shock data are not reported, as there were few differences on the whole between the pre- and postlesion weeks. The daily total lever pulls per schedule ( $3 \mathrm{~h}$ each per day) were tabulated for each animal. The units of ileum motility were collated for each of the four avoidance schedules and for the rest periods to give five raw data points per animal per day. Each ileum data point was also transformed to a percent of the respective daily total of integrated units for each animal (Lockard, Ehle, \&
Foltz, 1972; Lockard et al., 1977). The transformation to percent equates daily ileum-motility output and allows comparisons among monkeys.

\section{Brain Lesions}

Differences in avoidance and ileum data were found between the cingulum- and control-lesion groups, but none of the differences among the cingulum animals were directly attributable to extent of cingulum ablation (see Lockard et al., 1977).

\section{Pilot Monkey No. 715}

To illustrate the data gathered in this study, the total daily lever pulls per schedule for monkey 715 are shown in Figure 2. (The avoidance conditioning programs 2,7 , and 12 occurred on the 3 weekends, respectively, for 3 days each, Friday, Saturday, and Sunday.) For this initial animal, the lever-pull totals of each of its seven schedules were for periods of $1.5 \mathrm{~h}$. Inspection of the curves reveals considerably more prelesion pulling (Figure 2, left side) on the four schedules with unavoidable shock $(3,5,6$, and 7). As would be expected, the least pulling was on passive avoidance (2), with active avoidance plus passive avoidance (4) and active avoidance alone (1) next.

On the whole, monkey 715 pulled noticeably less postcingulumotomy (Figure 2, right side). Initially, all of the curves except passive avoidance alone (2) had converged. However, the rate of lever pulling on the four schedules with active avoidance $(1,4,5$, and 7) was sufficient to avoid all but an occasional shock.

On the basis of this initial animal's performance, schedules 2, 3, and 6 (see Figure 2) were eliminated from the main experimental design and time on schedules $1,4,5$, and 7 was increased from 1.5 to $3 \mathrm{~h}$ each per day to increase the discriminability of these schedules. Moreover, since the prelesion avoidance rates of this monkey had essentially leveled off the 2 nd week, the number of avoidanceconditioning days was decreased from 18 to 14 , preand postlesion for all other animals.

\section{Avoidance Behavior Differences Between Lesion Groups}

Since the avoidance curves of most monkeys of the main study were very similar in pattern to those of pilot monkey 715 (Figure 2), they are not shown. In accord with No. 715's data, considerably more lever pulling on the unavoidable shock schedules (5 and 7) was exhibited by the animals during the prelesion period. As predicted, the uniformity postlesion of the cingulum monkeys was analogous to that of No. 715 (Figure 2), whereas the controllesion animals were considerably more variable in their postlesion avoidance behavior.

More specifically, the mean daily lever pulls of the 


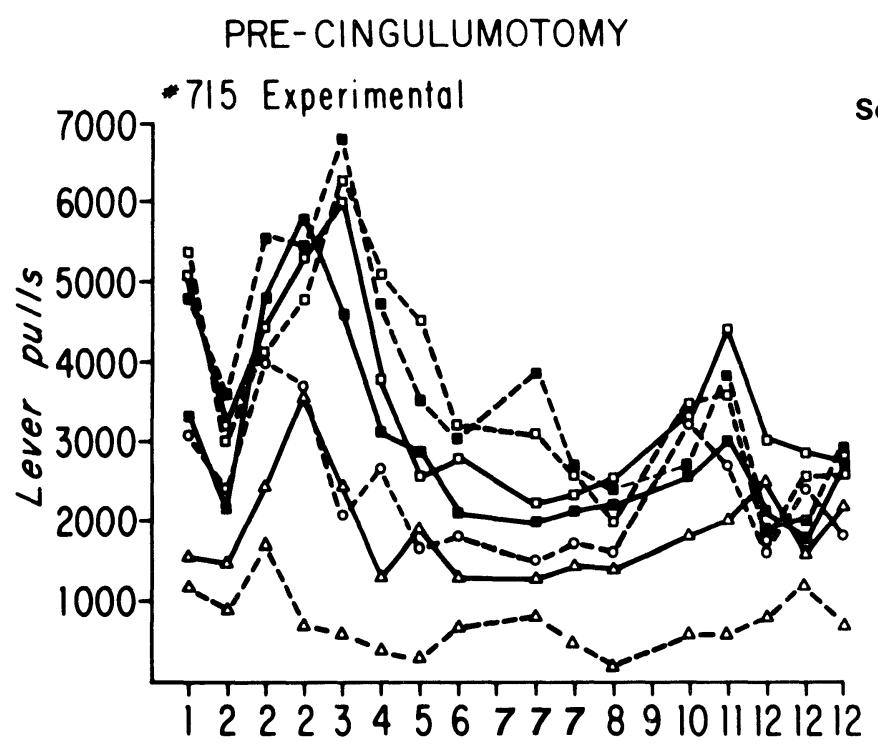

\section{POST-CINGULUMOTOMY}

Schedule 1 AA, Active avoidance $0--0$

2 PA, Passive avoidance $\Delta--\Delta$

3 UAS, Unavoidable shock $0--0$

4 AA+PA

5 AA+UAS

6 PA+UAS

7 AA+PA+UAS
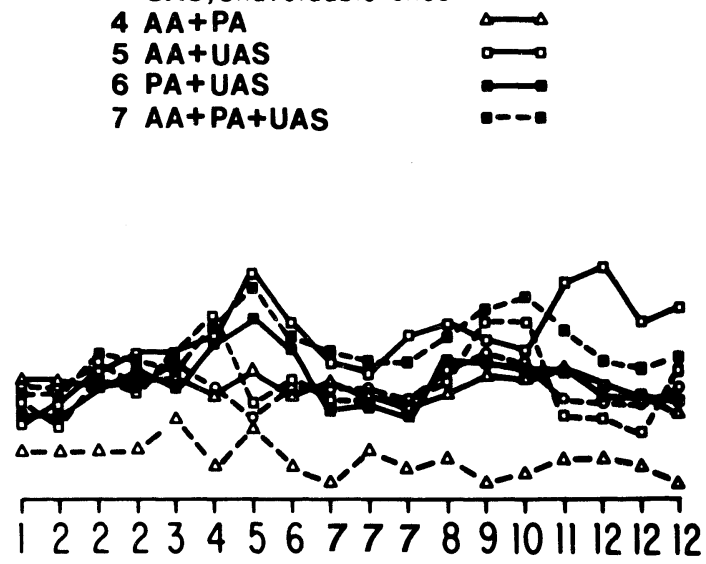

Avoidance-conditioning programs

Figure 2. Lever pulling of pilot, experimental monkey 715 during different avoidance schedules pre- and postcingulumotomy. Each schedule was presented for a total of $1.5 \mathrm{~h}$ per program per day.

cingulum- and control-lesion animals for the week before and the week after lesioning are given in Table 1. For purposes of simplicity of data presentation, the lever-pulling data of the 1 st week prelesion and the 2 nd week postlesion are not shown as they add little more to the comparison of the lesion groups. Those schedules with unavoidable shock ( 5 and 7 ) will be the primary focus, since the data of pilot monkey 715 suggested that they were the ones most likely to reveal differences in emotionality. Cingulum animal 659 showed a disproportionate drop in mean pulls after lesioning on Schedule 5 $(\mathrm{p}<.001)$ and Schedule $7(\mathrm{p}<.02)$. The mean lever pulls of cingulum monkey 658 increased considerably ( $p<.001$ ) for schedules 5 and 7 (and, to a lesser extent, for schedules 1 and 4 as well) the first post-

Table 1

Mean Daily Lever Pulls and Relevant Differences Among Avoidance Schedules Before and After Control and Cingulum Lesions

\begin{tabular}{|c|c|c|c|c|c|c|c|c|}
\hline \multirow[b]{3}{*}{ Animal } & \multicolumn{8}{|c|}{ Schedule } \\
\hline & \multicolumn{4}{|c|}{ Prelesion (2nd Week) } & \multicolumn{4}{|c|}{ Postlesion (1st Week) } \\
\hline & 1 & 4 & 5 & 7 & 1 & 4 & 5 & 7 \\
\hline \multicolumn{9}{|c|}{ Cingulum-Lesion Group: Experimental } \\
\hline 716 & 8,760 & 6,230 & 12,670 & 8,690 & 10,740 & 6,480 & 12,320 & 8,690 \\
\hline 659 & 6,900 & 4,638 & $7,523 *$ & $4,433 * *$ & 3,183 & 2,917 & $3,930 \downarrow^{*}$ & $3,445 \downarrow * *$ \\
\hline 658 & 2,905 & 2,992 & $4,170^{*}$ & $3,929 *$ & 6,398 & 6,935 & $11,732 \uparrow *$ & $9,128 \uparrow *$ \\
\hline 140 & 7,941 & 7,996 & 9,109 & 9,209 & 6,407 & 8,365 & 7,405 & 8,254 \\
\hline \multicolumn{9}{|c|}{ Cingulum-Lesion Group: Yoked } \\
\hline 717 & 550 & 340 & $3,510^{*}$ & $1,700 \dagger$ & 720 & 600 & $810 \downarrow *$ & $990 \downarrow \dagger$ \\
\hline 672 & 47 & 112 & 258 & 273 & 17 & 10 & 80 & 45 \\
\hline 180 & 504 & 229 & 931 & 287 & 911 & 377 & 1,262 & 621 \\
\hline \multicolumn{9}{|c|}{ Control-Lesion Group: Experimental } \\
\hline 863 & 29,968 & 30,767 & 41,023 & 38,414 & 26,691 & 26,842 & 9,219 & 26,828 \\
\hline 640 & 13,977 & 14,899 & 18,485 & 18,066 & 4,510 & 6,046 & 7,970 & 8,655 \\
\hline 116 & 5,804 & 5,162 & 7,098 & 6,222 & 5,196 & 4,652 & 5,548 & 5,143 \\
\hline \multicolumn{9}{|c|}{ Control-Lesion Group: Yoked } \\
\hline 639 & 7 & 30 & 8 & 133 & 3 & 4 & 9 & 12 \\
\hline 210 & 1,130 & 30 & 1,577 & 194 & 661 & 29 & 1,931 & 174 \\
\hline
\end{tabular}

Note-Relevant significant differences among the avoidance schedules are indicated by probability levels of t test (two-tailed) values. An arrow in the upper right corner of some data cells indicates the direction of significant change in lever-pulling performance in comparison to corresponding prelesion data. $\quad{ }^{*} p<.001 . \quad{ }^{* *} p<.02 . \quad t_{p}<.05$. 
Table 2

Mean Percent Ileum Motility and Relevant Differences Among Avoidance Schedules, Before and After Control and Cingulum Lesions

\begin{tabular}{|c|c|c|c|c|c|c|c|c|c|c|c|}
\hline \multirow[b]{3}{*}{ Animal } & \multicolumn{10}{|c|}{ Schedule } & \multirow{3}{*}{$\begin{array}{c}\text { Prelesion Ileum } \\
\text { Motility Stress } \\
\text { Response }\end{array}$} \\
\hline & \multicolumn{5}{|c|}{ Prelesion } & \multicolumn{5}{|c|}{ Postlesion } & \\
\hline & 1 & 4 & 5 & 7 & Rest & 1 & 4 & 5 & 7 & Rest & \\
\hline \multicolumn{12}{|c|}{ Cingulum-Lesion Group: Experimental } \\
\hline 716 & 9 & 17 & 16 & 11 & 46 & & & & & & Hypermotile \\
\hline 659 & 11 & 11 & $19 * *$ & 14 & 45 & 11 & 13 & $12 \downarrow * *$ & 13 & 50 & Hypomotile \\
\hline 658 & 23 & 11 & 10 & $11 \dagger \dagger$ & 45 & 12 & 10 & 13 & $19 \uparrow \dagger \dagger$ & 47 & Hypermotile \\
\hline 140 & 14 & 7 & $11 \dagger$ & $12^{* *}$ & 55 & 16 & 12 & $17 \uparrow \dagger$ & $18 \uparrow * *$ & 37 & Hypermotile \\
\hline \multicolumn{12}{|c|}{ Cingulum-Lesion Group: Yoked } \\
\hline 717 & 11 & 15 & 10 & 12 & 52 & 11 & 8 & 10 & 12 & 59 & Ambiguous \\
\hline 672 & 5 & 10 & 22 & 22 & 42 & & & & & & No Change \\
\hline 180 & 14 & 11 & 15 & 15 & 45 & & & & & & Hypermotile \\
\hline \multicolumn{12}{|c|}{ Control-Lesion Group: Experimental } \\
\hline 863 & 5 & 14 & 13 & 19 & 50 & 8 & 15 & 17 & 24 & 35 & Hypomotile \\
\hline 640 & 13 & 12 & 15 & 13 & 47 & 15 & 15 & 11 & 13 & 46 & Hypermotile \\
\hline 116 & 10 & 16 & 12 & 10 & 52 & 11 & 11 & 13 & 11 & 55 & Hypermotile \\
\hline \multicolumn{12}{|c|}{ Control-Lesion Group: Yoked } \\
\hline 639 & 22 & 7 & 13 & $10^{*}$ & 48 & 17 & 15 & 11 & $19 \uparrow^{*}$ & 39 & Hypermotile \\
\hline 210 & 8 & 5 & 14 & $17^{*}$ & 56 & 10 & 11 & 16 & $9 \downarrow *$ & 53 & Hypomotile \\
\hline
\end{tabular}

Note-Relevant significant differences among the avoidance schedules are indicated by probability levels of $t$ test (two-tailed) values. An arrow in the upper right corner of some data cells indicates the direction of significant change in percent motility in comparison to corresponding prelesion data. The prelesion total daily ileum motility response to stress-conditioning (i.e., a combination of the avoidance schedules) for each monkey is classified in the right column. ${ }^{*} p<.001 . \quad{ }^{* *} p<.01 . \quad$ tp $<.02 . \quad$ †tp $<.10$ (n.s.).

lesion week. Animals 716 and 140 showed no unusual postlesion changes in mean lever pulling on any schedule. However, yoked cingulum animal 717 decreased significantly its lever pulling on schedules 5 $(\mathrm{p}<.001)$ and $7(\mathrm{p}<.05)$. [A decrease on all four avoidance schedules (i.e., extinction; e.g., Nos. 672 and 639) or an increase on all schedules (i.e., reminiscence; e.g., No. 180) is what would be expected (all else equal) of the yoked monkeys the first few days postlesion.] In contrast to the cingulum group, no significant differences in mean lever pulls were found for any of the control-lesion animals on schedules 5 and 7 (as well as schedules 1 and 4).

\section{Comparisons of Percent Ileum Motility and a Prelesion Stress-Response Classification}

The mean ileum motility with respect to the different avoidance schedules, pre- and postlesions, for all monkeys are shown in Table 2 . Theoretically, the mean percent ileum data of each animal for each schedule should equal one another $(121 / 2 \%)$, the total of which $(50 \%)$ should equal the mean percent for rest $(50 \%)$, since $12 \mathrm{~h}$ per day were spent on the avoidance schedules and rest, respectively. Statistical comparisons are shown in Table 2, where the percent motility of the unavoidable-shock schedules (5 and 7) noticeably deviated from equality.

Since the present authors previously found (Lockard et al., 1977) that cingulum ablations tended to reverse the total daily ileum motility response (called prelesion stress response in Table 2 , right column) to avoidance conditioning, it is interesting to note that the direction of postlesion percent motility changes on the unavoidable shock schedules was, instead, consistent with that classification. In other words, No. 659 was hypomotile initially in its total daily ileum output and reduced its postlesion percent motility on schedule 5. Monkeys 658 and 140 were classified as hypermotile in their prelesion total daily ileum output and also increased their postlesion percent motility on schedules 5 and 7. Monkey 717's classification was somewhat ambiguous (Lockard et al., 1977) and its percent motility on the unavoidable shock schedules ( 5 and 7 ) did not change postlesion.

The above observation was also true for two yoked control-lesion animals (Table 2, bottom); hypermotile monkey 639 and hypomotile monkey 210 increased and decreased, respectively, their percent ileum motility on schedule 7 postlesion. However, experimental, control-lesion animals 640 and 116 , both hypermotile, manifested no unusual postlesion changes in percent motility on the unavoidable shock schedules; and hypomotile monkey 863 increased its already unusually high motility on schedule 7.

\section{Comparisons of Percent Ileum Motility and Avoidance Behavior}

If the data of Tables 1 and 2 are compared, it is found that for the same animal the motility and avoidance postlesion changes are often in the same 
direction. For cingulum experimental animal 659, its ileum motility during schedule 5 decreased postlesion with a decrease in lever pulling on that schedule. Similarly, No. 658 simultaneously increased its percent ileum motility and lever pulling on postlesion schedule 7. Monkey 140 lever pulled consistently more postlesion on schedules 5 and 7 , and its percent gut motility also increased on those same schedules. Yoked monkey 717 was inconsistent; there were no significant changes postlesion in its percent ileum motility on schedules 5 and 7 , whereas there were differences in lever pulling behavior.

Similar to cingulum animal 659 , experimental control-lesion animal 863 (Table 2, middle) was hypomotile in its total daily ileum motility during avoidance conditioning (e.g., Figure 1) and was hypermotile during prelesion schedule 7 . Unlike No. 659, however, monkey 863 was also hypermotile during postlesion schedule 7. Animals 640 and 116 showed no appreciable ileum motility (Table 2) or avoidance differences (Table 1) postlesion. Although among control-lesion animals no statistical lever-pulling changes were manifested (Table 1), two control-lesion animals (monkeys 639 and 210) showed several ileum motility changes (Table 2, bottom) that were consistent with their prelesion stress response of hypermotile and hypomotile, respectively.

\section{DISCUSSION}

The data indicate that: (a) The monkeys consistently lever pulled more (even some of the yoked controls) on avoidance shcedules with occasional unavoidable shock and less on a mixed schedule of active plus passive avoidance. (b) Whereas the daily ileum-motility patterns (namely, from day to day) of these animals did not change during avoidance conditioning (see Lockard et al., 1977), the percent motility per avoidance schedule within a day did differ. (c) The lever pulling and percent ileum motility per avoidance schedule of these monkeys largely paralleled one another; if either changed, often the other changed in a similar direction. (d) Cingulum ablations seemed to either augment or reduce both avoidance behavior and percent ileum motility, depending upon the particular monkey. (e) The direction of change in avoidance behavior and percent motility postcingulumotomy seemed to be related to the animal's characteristic ileum response (hypo- or hypermotile) during prelesion avoidance conditioning.

The monkeys, in general, lever pulled considerably more on the avoidance schedules with occasional unavoidable shock (schedules 5 and 7). This phenomenon was manifested even after extinction (yoked animals) or after attenuation of avoidance behavior by cingulumotomy. These findings are consistent with other avoidance data employing random unavoidable shock (Rescorla, 1966; Rescorla \& Solomon, 1967; see also Dubois \& Natelson, 1978). On the whole, the animals also pulled least on the active plus passive avoidance schedule (4). Both results are interesting when contrasted with what would be expected theoretically: Since each schedule was composed of active avoidance, with the same time and intensity settings, the most efficient rate of lever pulling would be the same for all schedules. Empirically, the monkeys did not perform that way, at least in the time period used in this study.

Nevertheless, no conclusion can be drawn about monkey emotionality from just the lever-pulling data alone, since the data on the unavoidable shock schedules postlesion could be logically attributed to poor or regressed discrimination among the schedules by the animals. The parallels between the percent ileum motility and the avoidance data on the unavoidable shock schedules (if it can be assumed that the former data are not a direct consequence of the latter data, or vice versa) do suggest that the animals were more emotional on those schedules. For instance, before lesioning, cingulum monkeys (e.g., No. 659) initially classified as hypomotile (i.e., statistically less total motility during stress days than control days; see Figure 1, left side) lever pulled the most on schedules 5 and/or 7 and their percent ileum motility was highest during those schedules (compare Tables 1 and 2). Postcingulumotomy, these animals pulled considerably less on those schedules and their percent motility significantly decreased. Similarly, but in the opposite direction, monkeys (e.g., No. 658) initially classified as hypermotile (i.e., significantly more total motility during stress days than control days; see Figure 1, left side), lever pulled more postcingulumotomy than before lesioning on schedules 5 and/or 7, and their percent ileum motility also noticeably increased. If the above hypothesis is valid, it would seem that in terms of daily total motility, cingulum ablation reduced the emotionality of hyperresponders and augmented the emotionality of hyporesponders (as reported by Lockard et al., 1977), but for particularly aversive schedules (i.e., 5 and/or 7), the animals behaved postcingulumotomy consistent with their premotility classification.

The difference in responses from animal to animal, both in lever pulling and gut-motility following the limbic lesion of cingulumotomy, indicates again the necessity of improved animal classification in order to start experimental comparisons with animals which are similar. Clinical experiences have already indicated that basic personality differences are related to treatment results obtained in cingulumotomy and that patient selection is critical in achieving good prognosis (Foltz \& White, 1966). In an earlier series of 12 paired monkeys who underwent cingulumotomy, pair-dominance behavior also was correlated with the pre-lesion emotional behavior clas- 
sification of the animals (Foltz, unpublished data). The present study supports those previous observations, and indicates that consistent or even predicted responses (lever pulling and gut motility) in these animals following cingulum lesions will not be attained until preexperimental animals can be classified in the same way on the basis of emotional behavior.

Another interesting observation concerns the daily ileum motility patterns of rhesus monkeys. In previous studies by the present authors (Lockard et al., 1972, 1977; Haller, Lockard, \& Foltz, 1976), no change was found in these patterns during avoidance conditioning. The daily patterns were based upon the hourly percent of the total daily motility irrespective of the occurrences of the avoidance schedules. However, when the data are collated with respect to particular avoidance schedules and transformed to a percent of the daily total, as was done in the current study, changes in percent ileum motility are found. In obtaining the daily motility patterns in the previous studies, the motility changes as a function of schedule were equally distributed in the mean hourly percent as a consequence of counterbalancing by experimental design the occurrences of each (i.e., every 15-min avoidance schedule immediately preceded and followed every other one, at least once). When such balancing is done, the consistency of the daily ileum motility pattern can be taken as a measure of both the stability of an animal's ileum response and the adequacy of the design of the study.

\section{REFERENCES}

Barensten, R. I., \& Lockard, J. S. An automated multichannel programming, data collection and printout system for avoidance and stress conditioning of monkeys. Behavioral Research Methods \& Instruments, 1969, 1, 187-189.

Brady, J. V., Porter, R. W., Conrad, D. G., \& Mason, J. W. Avoidance behavior and the development of gastroduodenal ulcers. Journal of the Experimental Analysis of Behavior, 1958, 1, 69-72.

Dubois, A., \& Natelson, B. H. Habituation of gastric function suppression in monkeys after repeated free-operant avoidance sessions. Physiological Psychology, 1978, 6, 524-528.

Fisher, R. A., \& Yates, F. Statistical tables for biological, agricultural and medical research. New York: Hafner, 1963.

FolTz, E. L. Neurophysiological mechanisms in production of gastrointestinal ulcers. Journal of the American Medical Association, 1965, 187, 413-417.

Foltz, E. L., \& White, L. E., Jr. Rostral cingulumotomy and pain "relief." In R. S. Knighton \& P. R. Dumke (Eds.), Pain. New York: Little, Brown, 1966.

Haller, R. G., Lockard, J. S., \& Foltz, E. L. Avoidance behavior and ileum motility post-cingulumotomy in monkeys. Biological Psychiatry, 1976, 11, 175-193.

LOCKARD, J. S. Shaping avoidance behavior in restrained monkeys. Journal of the Experimental Analysis of Behavior, $1969,12,649-652$.

Lockard, J. S., Ehle, A. L., \& Foltz, E. L. Diurnal variation of ileum motility in monkey during different feedings and avoidance-conditioning schedules. Physiology \& Behavior, $1972,8,195-200$.

Lockard, J. S., Foltz, E. L., Ehle, A. L., \& Haller, R. G. Ileum motility during avoidance conditioning in monkey, preand post-cingulumotomy. Physiology \& Behavior, 1977, 18, 111-117.

Maclean, P. D. The triune brain, emotion, and scientific bias. In F. O. Schmitt (Ed.), The neurosciences, second study program. New York: The Rockefeller University Press, 1970.

Kescorla, R. A. Predictability and number of pairings in Pavlovian fear conditioning. Psychonomic Science, 1966, 4, 383-384.

Rescorla, R. A., \& Solomon, R. L. Two-process learning theory: Relationships between Pavlovian conditioning and instrumental learning. Psychology Review, 1967, 74, 151-182.

(Received for publication January 3, 1979; revision accepted December 13, 1979.) 